\title{
ASTEROSEISMOLOGY IN ACTION: PULSATING HOT WHITE DWARFS
}

\author{
STEVEN D. KAWALER \\ Iowa State University \\ Department of Physics and Astronomy \\ Ames, IA 50011 USA
}

\section{Introduction}

The development of helioseismology as a forefront area of solar and stellar astronomy has been fascinating to watch. Helioseismology takes full advantage of the benefits of lots of photons and a spatially resolved target. These allow measurement of tiny amplitude photospheric velocity and flux variations, and the precise identification of the oscillation modes corresponding to each observed frequency.

The challenges of stellar (as opposed to solar) seismology include the much smaller fluxes from stars and the lack of spatial resolution. These combine to severely limit the number of modes that are available for detailed study - in most cases, stars show no measurable variations! For stars, oscillations must be of much larger amplitude than seen in the Sun. In addition, the signal from high angular degree modes are wiped out by simple geometric cancellation in the disk-integrated measurements of stars. Thus, while solar seismology involves the study of millions of independent oscillation modes, a "rich" stellar pulsator shows a few dozen modes at best.

Asteroseismology can currently be divided into two broad areas - let's call them "virtual" asteroseismology and "active" asteroseismology" Active asteroseismology involves study of known nonradial pulsators - including the pulsating white dwarfs, $\delta$ Scuti stars, rapidly oscillating Ap stars, and others that have been discussed in this meeting. In this review, I'd like to illustrate a mature example of active asteroseismology by briefly recounting some of the successes of seismic investigations of pre-white dwarf stars. The ZZ Ceti stars fall outside the scope of this short review, but see the paper by Clemens (1997) in this volume, the review by Winget (1988), and papers by Brassard et al. (1992) and Bradley \& Winget (1991) for a flavor of what is happening there.

\subsection{WHY CARE ABOUT WHITE DWARFS?}

White dwarfs represent the final stage of evolution for most stars, including our own Sun. Locked within their interiors is their prior evolutionary history, and in particular the story of their last nuclear evolutionary stage. By "excavating" the surface layers of 
a white dwarf, we can determine the circumstances under which its progenitor left the asymptotic giant branch (AGB). The amounts of hydrogen (if any) and helium that remain tell the phase (either between or during thermal pulses) at which the parent AGB star expelled a planetary nebula. In addition, determination of the helium (and hydrogen) layer masses of white dwarf stars clarifies one of the important inputs in white dwarf cooling theory, reducing the uncertainty in the age of the galactic disk as determined through the white dwarf luminosity function.

In addition to the hot white dwarfs, cool pulsating white dwarfs (the ZZ Ceti stars) also place tight constraints on the internal structure of older white dwarf stars. One of the most exciting new areas of work on cool white dwarfs is the prospect of measuring properties of the crystalline interior of a massive ZZ Ceti star, as discussed in Winget et al. (1997). For a recent general review of the properties, evolution, and seismology of white dwarf stars, see the review by Kawaler (1996).

\subsection{THE WHOLE EARTH TELESCOPE}

Asteroseismological studies of white dwarfs provide constraints ranging from the hot pre-white dwarf PG 1159 stars to the cooler hydrogen-rich ZZ Ceti stars. Exciting results for white dwarfs include measurements of stellar masses, distances, rotation rates (both surface and interior), and internal stratification of composition. Seismological data has demonstrated empirically the action of gravitational settling in white dwarf envelopes, and provided measurements and important upper limits to the cooling rates of white dwarf stars. These are described briefly in the next section. Before discussing these results, some background on the observational aspects of white dwarf seismology are in order.

The principal instrument that is used for seismological observations of white dwarfs is the Whole Earth Telescope (WET). The Whole Earth Telescope is a unique instrument. It was developed by Ed Nather and Don Winget at the University of Texas in 1986, and over the past decade has developed into an active collaboration of over 50 scientists in 14 nations, and continues to produce many scientific results.

The success of the WET is due in large part to the vision of its founders as a collection of scientists working together on mutually interesting science. The common tool needed to make these various scientific inquiries is required to provide uninterrupted time-series photometry that with time-resolution of seconds over time scales of weeks; that is, observations are needed for 24 hours per day over many days. To accomplish this at a reasonable cost requires a network of observatories around the globe staffed by observers with a keen interest in the science - not simply telescope assistants, but active scientists.

Despite being composed of various types of telescopes at observatories of many nations around the globe, the WET is operated as a single instrument and thought of as such by those who participate in it. Target are selected through an informal proposal process. Members of the collaboration suggest targets to a WET committee (known as the "Council of the Wise") who then decide on the priority targets for upcoming observing runs. The PI for each selected target provides a written scientific justification, which is distributed to all collaboration members for use in preparing observing proposals for each of their sites (either locally operated, or relevant national observatories). If time is granted for an observer at a given site, then that site is added to the list of active sites for the run.

During the WET run, data are collected at each site using instruments that are 
functionally nearly identical: multichannel photoelectric photometers. These allow continuous monitoring of the target star, a comparison star, and a blank sky field. During a WET campaign, data are collected at a given site; following each night, the data are sent via e-mail to a central headquarters (typically in Ames, Iowa). This central HQ is staffed 24 hours/day by several WET astronomers as well as the principal investigator for the primary target. When data arrive at $\mathrm{HQ}$, they are immediately reduced and examined for any problems. After reduction, a given run is incorporated into the growing data set from all sites. If a problem is detected in a given observation, $\mathrm{HQ}$ personnel contact the observers (by telephone or e-mail) to discuss the issue and help arrive at a solution. This direct interactivity provides welcome support and encouragement to the observers in remote sites, and ensures high data quality across the network.

Following a run, the data are provided to the PI, who is responsible for final reduction and analysis, and for drafting the paper describing the results. Data rights reside with the PI through the initial publication, which is usually a period of 18 months or so. Authorship of this initial publication includes all participating observers and analysts. Subsequently, the data are made freely available to all who need it, both within and outside the collaboration.

\section{Past Results from Whole Earth Telescope Observations}

As many of the successes of white dwarf seismology have been studies of the hottest pulsating white dwarfs, I will largely confine this discussion to these stars: the pulsating central stars of planetary nebulae, and the pulsating PG 1159 stars.

\subsection{MASSES AND ROTATION RATES}

The observed oscillation modes in pulsating white dwarfs are high radial order $g$ modes of low degree (almost exclusively $l=1$ ). As a result, the mode periods follow the asymptotic formula

$$
P_{n l}=\frac{P_{o}}{\sqrt{l(l+1)}}(n+\epsilon) .
$$

This means that the periods of consecutive radial orders are equally spaced in period. This is the principal signature seen in the pulsation spectra of hot white dwarfs. The constant period spacing factor $P_{o}$ in these stars is primarily determined by the mass of the star (Kawaler \& Bradley 1994). Identification of the period spacing has been possible for three of the PG 1159 stars and one planetary nebula central star. The masses range from $0.55 M_{\odot}$ for the central star of NGC 1501 (Bond et al. 1996) up to $0.66 M_{\odot}$ for the coolest PG 1159 star PG 0122 (O'Brien et al. 1998).

As with solar $p$-modes, rotation can be deduced by identifying equal frequency splitting in the pulsation spectrum; a given mode may be split into $2 l+1$ components. The frequency splitting is given by the familiar expression

$$
\Delta \nu=\Omega\left(1-C_{n l}\right),
$$

if the star rotates as a solid body, where $C_{n l}$ is a parameter obtained for a given mode by an integral over the eigenfuctions. For white dwarfs, an additional simplification 
is that the splitting coefficient is, to a good approximation:

$$
C_{n l} \approx \frac{1}{l(l+1)}
$$

Identification of equally-spaced triplets in the pulsation spectrum therefore allows measurement of the rotation rate of a white dwarf. Also, the presence of triplets rather than quintuplets suggests that most modes are $l=1$, though several $l=2$ modes are seen in PG 1159. Rotation periods for white dwarfs range from 5 hours for PG 2131 (Kawaler et al. 1995) to 1.4 days for PG 1159 (Winget et al. 1991), with most being close to 1 day. Note that this mean rotation period of 1 day is not an artifact of diurnal aliases; the Whole Earth Telescope was designed to remove this effect.

\subsection{SUBSURFACE STRUCTURE}

When the mode spectrum is sufficiently rich, the period spacings can show regular departures from uniformity. This is the result of mode trapping by a subsurface composition discontinuity. Mode trapping allows us to determine the depth of this discontinuity, and therefore provides a key seismological tool for constraining the evolutionary history of white dwarfs.

As of now, one PG 1159 star and one DB pulsator have shown the clearest effects of mode trapping. PG 1159 itself was studied extensively by WET data (Winget et al. 1991). Kawaler \& Bradley (1994) showed that the period distribution could be matched by a model with a (helium-rich) surface layer of $3 \times 10^{-3} M_{\odot}$. Given the surface composition of PG 1159, this thin layer suggests that PG 1159 underwent a final thermal pulse early in its life as a white dwarf, and may have briefly returned to the AGB. Such a "born-again" scenario is plausible given the evolutionary calculations by Iben (1984) and others.

The other star with a measured subsurface composition transition is the pulsating DB white dwarf GD 358. Bradley \& Winget (1994) show that the pulsation periods observed by WET (Winget et al. 1994) are consisted with a model that has a pure helium layer with a thickness of $10^{-6} M_{\odot}$. This is surprisingly thin, given the thickness of the surface layer of PG 1159, which represents its possible progenitor.

\subsection{IMPACT ON EVOLUTIONARY MODELS: DIFFUSION}

The apparently large difference between the helium layer masses of GD 358 and PG 1159 seems to preclude their having a common origin, with GD 358 being the descendent of PG 1159. In fact, however, the two stars may indeed share common ancestry and a direct evolutionary link. PG 1159 shows a surface that is helium-rich, but has almost as much carbon and oxygen in its outer layers. GD 358 has a nearly pure helium surface.

At the high surface gravities of white dwarfs (with $\log g$ of 7.5 to 8.0 ) diffusive processes act to drain heavy elements from the surface, thus purifying the outer layers. Though this has been "known" for years, direct evidence has been limited to explaining the trace abundances of heavy elements in white dwarf atmospheres.

Evolutionary calculations of white dwarfs that include diffusive processes have been explored by a number of researchers. However, nearly all are concerned with the abundances of DA (hydrogen-rich) white dwarfs at cooler temperatures, or with the trace metal abundances of hot white dwarf stars. Those that deal with DB white 
dwarfs had not been fully time-dependent calculations. Dehner (1996) explored the evolution of PG 1159 stars to low temperature including the effects of diffusion, and found that, as expected, the surface helium abundance increases rapidly with time. As the model cools, a pure helium layer collects at the surface, and proceeds downwards. Dehner (1996) and Dehner \& Kawaler (1995) found that this pure helium layer reached a thickness of $10^{-6} M_{\odot}$ when the model reached a temperature of $25,000 \mathrm{~K}$, which is nearly identical to the conditions deduced by Bradley \& Winget (1994) based on WET seismology. Dehner \& Kawaler (1996) showed that the pulsation spectrum of their evolutionary model provided a good fit to the observed pulsation periods in GD 358 as well.

Thus seismology of GD 358 demonstrates that the computational aspects of timedependent diffusion in white dwarfs accurately represent not only the surface chemical evolution of white dwarfs, but also match the interior chemical stratification.

\section{Future Directions}

Aside from ongoing work in the seismology of pre-white dwarfs of the kind indicated above, there are additional areas of study. Continued measurement of white dwarf masses, rotation rates, and compositional stratification will refine the theory of white dwarf formation and evolution, helping us to lift the veil of ignorance that has clouded the upper-left corner of the $\mathrm{H}-\mathrm{R}$ diagram.

Beyond this now almost traditional seismological work, there are several new directions that are being explored that use the tools of stellar seismology. This section briefly outlines a few of these new possible directions. Again in this section I concentrate on pre-white dwarf stars. Studies of the cooler DA and DB white dwarfs are equally rich and promising, but lack of space prevents more discussion of these objects.

\subsection{CENTRAL STARS OF PLANETARY NEBULAE}

The "naked" PG 1159 stars (those without observable nebulae today) were, earlier in their lives, the central stars of planetary nebulae. Indeed, several PNNs show similar spectral features to those seen in the naked PG 1159 stars: CIV and OVI in with emission cores, and so on. Some of these central stars are also nonradial pulsators. Most of them have been discovered as pulsators by Howard Bond and Robin Ciardullo and their collaborators (see Ciardullo \& Bond 1996). The pulsation periods in the PNNs are usually much longer than in the naked PG 1159 pulsators. To date, 8 PNNs are known nonradial pulsators. Periods range from about 700 seconds up to several thousand seconds in these stars. The prospect of studying the global properties of PNNs as well as their interior structure through asteroseismology is very exciting. These stars represent the immediate consequences of thermal pulsing on the Asymptotic Giant Branch, as well as the ejection of planetary nebulae. Anything we can learn about their interior structure through asteroseismology will provide very important constraints on this fleeting stage of stellar evolution.

So far, only one of the PNN variables has been fully investigated in an asteroseismological sense. Bond et al. (1996) obtained multisite CCD time-series photometry of the central star of NGC 1501. They were able to identify several modes, and used them to deduce a mean period spacing of 20 seconds. This in turn suggests that the 
mass of the central star of NGC 1501 is approximately $0.55 M_{\odot}$, and that it rotates with a period of approximately 1.15 days.

A characteristic of the pulsation spectra of the PNNs is that they are very variable; coherent pulsation modes appear and disappear on short time scales ranging from days to a few years. However, the frequencies of the modes that are manifest at any given time show consistency with a single underlying mode spectrum. Observing these stars over several seasons allows us to collect a "library" of modes, the ensemble being used to deduce the parameters of the spectrum such as the mean period spacing, and rotational splitting values. Similar behavior is seen in some ZZ Ceti stars. Understanding the amplitude instabilities of these stars is difficult, but may be related to the strong winds that are present in PNNs, or to the fact that very high overtone modes are present that are influenced by nonlinear near-surface phenomena.

Another area of mystery about these stars is an apparent correlation between the morphology of the nebula and the pulsation periods of the central star. Pulsating PNNs fall into to groups based on their periods: one group shows periods in excess of 1000 seconds, while the second group show periods that are substantially shorter (averaging 700-800 seconds). Examination of the nebulae themselves shows that the long-period central star are surrounded by fairly symmetric and uniform nebulae. Examples include NGC 246 and NGC 1501. Central stars with short periods are embedded in messy, asymmetric nebulae: examples are the nebula surrounding RX J2117 (Appleton et al. 1993), and NGC 2671. Why should such a correlation exist? Is it merely coincidental, or does the pulsation of the central star influence the nebular morphology? Is it the other way around (does the nebular morphology influence the pulsational instabilities)? Or is there a correlation with stellar mass? Answers to these questions awaits full seismological analysis of a significant fraction of the pulsating central stars.

\subsection{ROTATIONAL INVERSIONS}

For the richest of the pulsating white dwarfs, PG 1159 and GD 358, several multiplets corresponding to consecutive radial overtones are present in the observed pulsation spectrum. The amount of rotational splitting in general differs from multiplet to multiplet, suggesting that one can estimate the departure of the interior from solidbody rotation. Equation 2 is strictly true only when the rotation rate is constant throughout the interior. If differential rotation exists within a white dwarf, some modes will sample the interior differently than others, producing departures from uniform splittings from mode to mode. Indeed, Winget et al. (1994) suggest that the observed splittings in GD 358 indicate that the outer layers of this star rotate much faster (by a factor of nearly 2) than the inner regions.

Kawaler, et al. (1998) explored the possibilities of using the observed splittings to estimate the internal rotation profile of these two stars. Using inversion techniques and forward calculations, they show that even though the information provided by the pulsations of white dwarf stars is much less extensive than by solar oscillations. some pulsators are rich enough to provide useful constraints on their internal rotation.

The rotational splittings are sensitive to mode trapping: the phase between the rotational splitting and period spacings (as functions of period) allows an observational determination of the sign of any slope in the rotation rate with depth. PG 1159 shows the signature of a rotation curve which is faster at the center than at the surface. Further observations of this star by the Whole Earth Telescope are now available 
(Winget, private communication); least-squares analysis of these data, in combination with the first WET data on this star, should be able to test this result.

For the case of the pulsating DB white dwarf GD 358, Kawaler et al. (1998) find that the observed spacings can be reproduced, even only approximately, if the star has a rapidly rotating inner $(r / R<0.2)$ core, a slowly rotating outer layer, and a counterrotating midsection; clearly such a rotation curve is unphysical. Thus the earlier suggestion by Winget et al. (1994) is not borne out by the more detailed calculations; Kawaler et al. (1998) conclude that the observed splittings are not consistent with any reasonable internal rotation curve. Still, they conclude that further observations of this star may provide a more consistent description of its internal rotation profile. This work on rotational inversions, though inconclusive, demonstrates the potential of the technique if longer and higher-precision observations can be obtained.

\subsection{MEASURING NEUTRINO COOLING VIA SECULAR PERIOD CHANGES}

During the pre-white dwarf phase, evolutionary models indicate that neutrino cooling is the dominant source of energy loss for stars near the lower end of the instability strip occupied by the variable pre-white dwarfs. The fraction of the total luminosity contributed by neutrinos increases with increasing mass. Recent asteroseismological analysis of light curves of pulsating pre-white dwarfs near the low temperature bound of the instability strip indicate a trend toward higher masses with decreasing temperature for pulsators within the strip. Thus, O'Brien et al (1997) point out that the cool PG 1159 pulsators should show the largest effects of neutrino cooling. Since the evolutionary changes in these stars will be reflected in the pulsation periods, detection of secular period changes in these stars allows measurement of their cooling rates. Future measurements of secular changes in the period of the dominant modes in these cool, massive pre-white dwarfs can therefore constrain the neutrino emission taking place in their electron degenerate cores, and may provide the first experimental test of thermal neutrino production rates.

\section{Conclusions: the Interface Between Pusation and Evolution}

With the advantage of having many objects that show nonradial pulsations, white dwarf seismology has advanced to the point where asteroseismological results are now fully integrated into the mainstream of this area of stellar astrophysics. No longer are white dwarf researchers strictly classified as "pulsation types" and "evolution types" and "atmosphere types". Research efforts in white dwarf formation and evolution, as well as in the use of white dwarfs in other astrophysical contexts, routinely include contributions from all of these inputs.

In addition to this mainstreaming of white dwarf seismology, white dwarf pulsators may teach us a lot about the physics of stellar pulsation, and the hydrodynamics of stellar matter. Seismological probes have almost exclusively relied on comparison of observed pulsation frequencies with linear adiabatic pulsation calculations. However, the observed pulsations also show strong nonlinear effects including harmonics, power spectrum peaks at frequencies that are the sums of frequencies of large-amplitude "normal" modes, disappearance and reappearance of normal modes, and other forms of complex amplitude modulation. These nonlinear behaviors contain a wealth of information about the physics of these stars. But because of the many complexities 
of nonlinear analysis, research is still at an early stage in attempting to mine this information.

It is important to point out that though this brief review has concentrated on WET observations and their interpretations, coordinated network observations are only one approach to the problem. As shown by Clemens (1997), who has observed pulsating white dwarfs at Keck Observatory and with HST, as well as work by Fontaine and coworkers at the $\mathrm{CFH}$ telescope, large aperture studies can provide important complementary information. With large telescopes, Clemens (1997) shows that the spectrum of the pulsators responds to the nonradial pulsations of the atmosphere, allowing new measurements of things like the temperature amplitude of the pulsations. and perhaps allowing mode identifications. Fontaine and his group demonstrate that with high $\mathrm{S} / \mathrm{N}$, low-amplitude modes can be found that are hidden in network observations with smaller telescopes. These low amplitude modes may help fill in the pulsation spectra, allowing seismological analysis on more complete mode sets.

Finally, significant research is possible with moderate aperture single-site observations. Once a pulsation spectrum has been decoded, follow-up observations at a single site can extend the timing baseline. With such a baseline, phasing of stable observed modes can allow determinations of $d P / d t$, which in turn places interesting constraints on the evolutionary time scales of the pulsators. Also, for those objects that show modes that come and go on time scales of months, single-site observations can identify these occasional pulsations and help fill in the overall spectrum (see, for example, Kleinman et al. 1994, O'Brien et al. 1996, and Bond et al. 1996).

\section{Acknowledgements}

The author thanks the organizers of IAU Symposium 185 and the NASA Astrophysics Theory Program for their support.

\section{References}

Appleton, P.N., Kawaler, S.D., \& Eitter, J. 1993, AJ, 106, 1973

Bond, H., Kawaler, S., Ciardullo, R. et al. 1996, AJ, 112, 2699

Bradley, P.A. \& Winget, D.E., ApJSupp, 75, 463

Brassard, P., Fontaine, G., Wesemael, F., \& Hansen, C.J. 1992, ApJSupp, 80, 369

Ciardullo, R. \& Bond, H. 1996, AJ, 111, 2332

Clemens, J.C. 1997, these proceedings

Dehner, B.T. 1996, Ph. D. Thesis, Iowa State University

Dehner, B.T. \& Kawaler, S.D. 1995, ApJL, 445, L141

Iben, I. Jr. 1984, ApJ, 277, 333

Kawaler, S. 1996, in Stellar Remnants: Saas-Fee Advanced Course 25, ed. G. Meynet \& D. Schaerer (Berlin: Springer-Verlag), p. 1-95

Kawaler, S. \& Bradley, P. 1994, ApJ, 427, 415

Kawaler, S., Sekii, T., \& Gough, D.O. 1998, MNRAS, in press

Kawaler, S., et al. (the WET collaboration) 1995, ApJ, 450, 350

Kleinman, S. et al. (the WET collaboration) 1994, ApJ, 436, 875

O'Brien, M.S., Clemens, J.C., Kawaler, S.D., \& Dehner, B.T. 1996, ApJ, 467, 397

O'Brien, M.S. et al. (the WET collaboration) 1998, ApJ, in press

Winget, D.E. et al. (the WET collaboration) 1991, ApJ, 378, 326

Winget, D.E. et al. (the WET collaboration) 1994, ApJ, 430, 839

Winget, D.E., Kepler, S.O., Kanaan, A., Montgomery, M., \& Giovannini, O. 1997, ApJL. 487, L191 